\title{
Weak Signal Detection Based on Chaotic Oscillators
}

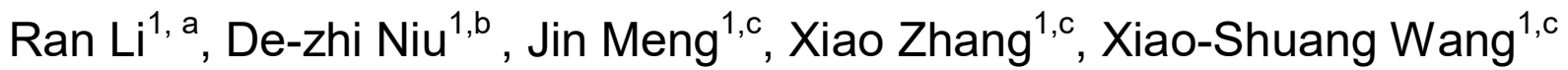 \\ ${ }^{1}$ College of Information and Communications, National University of Defense Technology, Xi'an, \\ Shaanxi, 710106, China

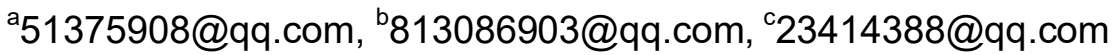

\begin{abstract}
This paper studies the implementation of weak signal detection chaotic oscillators. Also, the detection model of the system is theoretically studied and analyzed. The main mechanism of the chaotic oscillator detecting the same frequency signal and the non-frequency signal located in the difference frequency range is expounded. The dissertation provides a reference for the realization of weak signal detection using chaotic oscillator.
\end{abstract}

Keywords: Weak signal; Chaotic oscillator; Co-frequency signal; Non-co-frequency signal; Detection statistic

\section{Introduction}

Signal detection technology is fundamentally used in the field of engineering, especially weak signal detection under the background of strong noise, which is always a hot and difficult research at home and abroad. The traditional method is based on the linear and deterministic system so that the minimum detectable signal-to-noise ratio can be achieved is limited. Usually, when the signal to noise ratio is lower than $-10 \mathrm{~dB}$, the conventional detection method is difficult to extract from the noise background The characteristic parameters of the signal.

In practical situations, signal detection in many environments is low signal-to-noise ratio detection, such as signal detection in sea clutter background, detection of underwater target signal, signal detection in some spectrum sensing environments, weak signal detection in photoacoustic tank, etc. In traditional treatment, sea clutter is often filtered out as noise. But in fact, sea clutter, a useful signal, is an echo produced by the radar irradiating the sea surface. When the sea environment is complex, The generation of clutter is affected by waves, sea breeze, tides and so on, which can interfere with the data detected by sea radar and is difficult to detect. This is the so called weak signal detection problem in the background of sea clutter. In passive sonar signal processing, line spectrum detection and extraction play a decisive role, because the line spectrum can be used to estimate the target's motion parameters. When the target distance is far or the target signal is weak, the detection becomes more difficult. In addition, it is more important to detect underwater objects with low signal-to-noise ratio such as submarines with very high noise interference. Some signals in the spectrum sensing are abnormally weak due to the propagation distance or attenuation, etc., and it is difficult to detect the signal at the transmitting end. However, the local oscillator leakage of the receiving super heterodyne receiver can be used to detect whether there is another user with a certain frequency. The leakage signal of local oscillator under this circumstances is also very weak. The atmospheric pollution source gas content is so small that using photoacoustic spectroscopy to 
monitor the photoacoustic signal is extremely weak. Due to the impact of atmospheric noise, The size of the noise in the signal to be detected can be compared to itself, or even higher, which is a problem to be solved in the field of photoacoustic spectroscopy detection.

With the rapid development of nonlinear science and computer technology, the development of chaos theory, which is based on chaotic system for weak signal detection, provides a new technological means for weak signal detection.

Since Brix used Duffing oscillator to detect weak signals in 1992, chaos detection methods has been attracting more and more attention in theoretical research and engineering implementation, and has been applied to fields like optoelectronics, communication, radar, sonar, fault diagnosis and so on. According to the results of the existing literature, the minimum signal to noise ratio (SNR) of chaotic signal detected by chaotic oscillator has been detected to be about $-110 \mathrm{~dB}$, which also fully demonstrates the unique advantage of the oscillator in weak signal detection.

At present, typical methods of chaos detection mainly include weak signal detection based on Chen system, weak signal detection based on Rossler system, weak signal detection based on Lorenz system, and weak signal detection based on Duffing oscillator. These not only effectively combine the dynamic characteristics of chaotic system with signal processing, but also provide a new direction for the realization of weak signal detection]. But at the same time, it should be recognized that in these methods, Chen, Rossler and Lorenz systems are with three state output differential system, and their systems consist of different states of mixed cross terms. This structure not only caused the high complexity and low performance of the system, but made it more difficult to identify the rate of state change for signal detection when there is no Duffing system. Therefore, the weak signal detection based on Duffing oscillator, obtaining more attention, has become one of the most chaotic detection inspection representative measurement methods. Although the basic Duffing oscillator system has simple structure compared with other systems, its most direct characteristic is that when the weak signal is detected, the system state changes very obviously, and the system has the advantages of high sensitivity and strong noise immunity, etc. Moreover, the new Duffing oscillator obtained from the basic Duffing oscillator deformation or coupling has good detection results, which makes the signal detection methods develop rapidly.

\section{Main Principle of Detecting Weak Signals by Chaotic Oscillator}

Here, the main principle of detecting weak signals by chaotic oscillator is illustrated by an example of the basic Duffing oscillator. The basic Duffing equation is generally expressed as:

$$
\bar{x}+\mu \bar{x}-x+x^{3}=F \cos \left(w t^{\prime}\right)
$$

In this equation, $\mu$ is a damping ratio and $F \cos (w t)$ is the periodic strategy of the system. In the Duffing equation, the dynamic behavior of the adjoint strategy is $\mathrm{F}$, and the dynamic behavior of the system is rich, showing the periodic oscillation, the chaos and the large period state respectively. Mark the critical value between chaotic and periodic situation as Fd. so the mechanism using Duffing oscillator weak signal detection is: set the $\mathrm{F}$ as critical value, when adding the weak signal to be measured, the driving amplitude of the system will be greater than the total Fd, then the signal can be measured according to the state change of the system.

Let the signal to be measure as $\mathrm{s}(\mathrm{t})=\mathrm{A} \cos (\mathrm{wxt}+\varphi)=A \cos ((w+\Delta w) t+\varphi)($ where $\mathrm{wx}=\mathrm{w}+\Delta w)$, then the Duffing oscillator signal detection model is: 


$$
\bar{x}+\mu \dot{x}-x+x^{3}=F_{d} \cos (w t)+s(t)+n(t)
$$

Due to Duffing system has strong noise immunity, so the impact of noise can be ignored in a certain range of signal to noise ratio, and therefore the noise factor is also ignored in the coming discussion.

Thus, in Equation (2), the total system dynamics (composite dynamics) are:

$$
F_{d} \cos (w t)+s(t)=F_{d} \cos (w t)+A \cos ((w+\Delta w) t+\varphi)=F(t) \cos (\omega t+\theta(t))
$$

Among them, $\mathrm{F}(\mathrm{t})$ is the amplitude of the synthetic propulsion force and $\theta(t)$ is the initial phase of the synthetic propulsion force. The expressions are:

$$
\begin{gathered}
F(t)=\sqrt{F_{d}^{2}+2 F_{d} A \cos (\Delta w t+\varphi)+A^{2}} \\
\theta(t)=\arctan \frac{A \sin (\Delta \omega t+\varphi)}{F_{d}+A \cos (\Delta w t+\varphi)}
\end{gathered}
$$

For weak signals, $\mathrm{A}<<\mathrm{Fd}$, then $\theta(t)$ is approximately equal to 0 . In this way, weak signal detection based on Duffing oscillator is to discuss the relationship between $F(t)$ and $F d$, and determine the situation of the system to achieve the detection of the signal under test. Due to the existence of $\Delta w$, the signal detection will be divided into the same frequency or different frequency in two cases.

In addition, in order to detect any signal, an equivalent Duffing equation is often considered. The equivalent Duffing detection model (neglected $\theta(t)$ ) with the signal to be measured is:

$$
\ddot{x}+\mu w \bar{x}-w^{2} x+w^{2} x^{3}=w^{2}\left(F_{d} \cos (w t)+s(t)\right)=w^{2} F(t) \cos (w t)
$$

The corresponding equation is

$$
\left\{\begin{array}{l}
\dot{x}=w y \\
\dot{y}=w\left(-\mu y+x-x^{3}+F_{d} \cos (w t)+s(t)\right)
\end{array}\right.
$$

When the signal under test has the same frequency as the driving force, that is when $\Delta w=0$ :

$$
F(t)=\sqrt{F_{d}^{2}+2 F_{d} A \cos \varphi+A^{2}}
$$

According to the analysis, when $\varphi \in\left(0, \pi-\arccos \left(A /\left(2 F_{d}\right)\right)\right) \cup\left(\pi+\arccos \left(A /\left(2 F_{d}\right)\right), 2 \pi\right), \mathrm{F}(\mathrm{t})>\mathrm{Fd}$. In this case, the system will undergo chaotic state to large-cycle transformation (showing large periodic state), and the detected signal can be detected through this change. The angle interval corresponding to $\varphi$ at this moment is called detectable region. Also, when $\varphi \in\left[\pi-\arccos \left(A /\left(2 F_{d}\right)\right), \pi+\arccos \left(A /\left(2 F_{d}\right)\right)\right], \mathrm{F}(\mathrm{t}) \leq \mathrm{Fd}$. At his moment, the state of the system is still in a chaotic state. Although the signal under test is added, the signal can not be detected. That is, the signal under test falls into a blind area for detection, which is a problem to be solved in practice.

$$
\left(F_{d}-A\right)^{2} \leq F_{d}^{2}+2 F_{d} A \cos (\Delta \omega t+\varphi)+A^{2} \leq\left(F_{d}+A\right)^{2}
$$


$\mathrm{F}(\mathrm{t})$ will change inside $[\mathrm{Fd}-\mathrm{A}, \mathrm{Fd}+\mathrm{A}]$ when the system enters chaotic situation. Studies have shown that, when $\mid \Delta w / w \leq 0.03$, the regular intermittent chaos phenomenon can be identified, and the period of intermittent chaos in the time domain is $2 \pi / \Delta w$, then the signal to be measured can also be detected.

\section{Ackowledgment}

This work is supported by National natural science foundation youth project (No. 61701534)

\section{References}

[1] Birx D L. Chaotic oscillator and CMFFNS for signal detection in noise environments[C]. IEEE International Joint Conference on Neural Networks, Baltimore,USA,1992:821-888.

[2] Jean Chamberlain Chedjou and Kyandoghere Kyamakya. A Universal Concept Based on Cellular Neural Networks for Ultrafast and Flexible Solving of Differential Equations[J]. IEEE TRANSACTIONS ON NEURAL NETWORKS AND LEARNING SYSTEMS, 2015,26(4): 749-762.

[3] Hesan Vahedi, G. B. Gharehpetian,and Mehdi Karrari. Application of Duffing Oscillators for Passive Islanding Detection of Inverter-Based Distributed[J] IEEE TRANSACTIONS ON POWER DELIVERY, 2012,27(4):1973-1983.

[4] Alexander Jimenez-Triana, Wallace Kit-Sang Tang, Guanrong Chen, etc. Chaos Control in Duffing System Using Impulsive Parametric Perturbations[J].IEEE TRANSACTIONS ON CIRCUITS AND SYSTEMS,2010,57(4):305-309.

[5] V.N. Patel,N. Tandon,R.K. Pandey. Defect detection in deep groove ball bearing in presence of external vibration using envelope analysis and Duffing oscillator[J]. Measurement, 2012,45:960-970.

[6] Xu Yan-Chun, Yang Chun-Ling, Qu Xiao-Dong. A new approach to detecting weak signal in strong noise based on chaos system control[J]. Chinese Physics B,2010,19(3):030516:1-5.

[7] SHI Si-Hong, YUAN Yong, WANG Hui-Qi, LUO Mao-Kang. Weak Signal Frequency Detection Method Based on Generalized Duffing Oscillator[J].CHIN. PHYS. LETT., 2011,28(4):040502 1-4.

[8] YUAN Peixin, LI Yongqiang. Approximate Solutions of Primary Resonance for Forced Duffing Equation by Means of the Homotopy Analysis Method[J]. CHINESE JOURNAL OF MECHANICAL ENGINEERING， 2011,24(3):501-506.

[9] Gui-tian HE, Mao-kang LUO. Dynamic behavior of fractional order Duffing chaotic system and its synchronization via singly active control[J]. Applied Mathematics and Mechanics(English Edition),2012, 33(5): 567-582. 